\title{
Construction of ground control points for spacecraft using optimization-simulation model
}

\author{
I.N. Kartsan \\ Marine Hydrophysical Institute, Russian Academy of Sciences, 2, Kapitanskaya St., Sevastopol, \\ 299011, Russian Federation \\ Reshetnev Siberian State University of Science and Technology, 31, Krasnoyarsky Rabochy \\ Av., Krasnoyarsk, 660037, Russian Federation \\ Sevastopol State University, 33, Universitetskaya St., Sevastopol, 299053, Russian Federation \\ FGBNU «Expert-Analytical Center», 33/4 Talalikhina St., Moscow, 109316, Russian Federation \\ E-mail: kartsan2003@mail.ru
}

\begin{abstract}
The article deals with the application of simulation modeling and optimization-simulation approach to solving problems of synthesis of structures of automated spacecraft control systems, namely, the choice of spacecraft control points, which are multifunctional aggregates of stationary and mobile elements dispersed in space with developed technical means of receiving, transmitting and processing information. Various approaches to the joint use of optimization and simulation models in the synthesis of the structure of complex systems are analyzed. The main attention is paid to the possibility of joint use in the synthesis of optimization and simulation models, their rational interaction in optimization and simulation procedures that describe both the composition and interrelationships of the structural elements of the system, as well as dynamic and stochastic aspects of their functioning. An optimization-simulation approach based on the joint use of optimization and simulation models in the process of searching for optimal structure options is proposed. Rational mapping of a set of interrelated functions performed by a control system into a set of interconnected nodes with appropriate technical means, taking into account the costs of creating or reconstructing the system, the costs of operation and operation, the requirements of operational efficiency of management, the reliability of technical means, the survivability and globality of the control system and other characteristics. Consideration of the problem of optimizing the distribution of tasks included in the control loop by levels and control nodes of the system and determining a set of technical means that minimize the costs of equipping nodes with technical means and their operation when performing restrictions on efficiency, hardware reliability of performing control tasks, weight and energy consumption of on-board equipment, loading of nodes, etc.
\end{abstract}

Keywords: optimization and simulation approach, simulation modeling, algorithm, model, spacecraft, a network of ground control points

Acknowledgements: This study was supported by the Russian Federation State Task № 0555-2021-0005. This work was carried out within the framework of the state assignment of the Ministry of Education and Science of the Russian Federation on "Conceptual modeling of the information and educational environment of human capital reproduction in a digital economy" (Cipher FNRN - E). This work was performed within the framework of the state assignment of the Ministry of Education and Science of Russia on the topic "Development of new methods of autonomous navigation of spacecraft in outer space" (Cipher FNRN - S). 
УДК 004.021

\title{
Построение наземных пунктов управления космическими аппаратами с использованием оптимизационно-имитационной модели
}

\author{
И.Н. Карцан \\ Морской гидрофизический институт РАН, ул. Капитанская, 2, г. Севастополь, 299011, \\ Российская Федерация, \\ Сибирский государственный университет науки и технологий имени академика М.Ф. \\ Решетнева, пр. Красноярский рабочий, 31, Красноярск, 660037, Российская Федерация \\ Севастопольский государственный университет, ул. Университетская, 33, г. Севастополь, \\ 299053, Российская Федерация, \\ ФГБНУ «Экспертно-аналитический центр», ул. Талаллихина, 33/4, г. Москва, 109316, \\ Российская Федерация \\ E-mail: kartsan2003@mail.ru
}

\begin{abstract}
Аннотация. Рассматриваются вопросы применения имитационного моделирования и оптимизационноимитационного подхода к решению задач синтеза структур автоматизированных систем управления космическими аппаратами, а именно, выбора пунктов управления космическими аппаратами, которые представляют собой рассредоточенные в пространстве многофункциональные совокупности стационарных и подвижных элементов с развитыми техническими средствами приема, передачи и обработки информации. Анализируются различные подходы к совместному использованию оптимизационных и имитационных моделей при синтезе структуры сложных систем. Основное внимание уделяется возможности совместного использования в процессе синтеза оптимизационных и имитационных моделей, их рациональное взаимодействие в оптимизационно-имитационных процедурах, описывающих как состав и взаимосвязи структурных элементов системы, так и динамические и стохастические аспекты их функционирования. Предложен оптимизационно-имитационный подход, основанный на совместном использовании оптимизационных и имитационных моделей в процессе поиска оптимальных вариантов структуры. Рациональное отображение множества взаимосвязанных функций, выполняемых системой управления, на множество взаимосвязанных узлов с соответствующими техническими средствами с учетом затрат на создание или реконструкцию системы, затрат на эксплуатацию и функционирование, требований оперативности управления, надежности технических средств, живучести и глобальности системы управления и других характеристик. Рассмотрение проблемы оптимизации распределения задач, входящих в контур управления по уровням и управляющим узлам системы и определении набора технических средств, минимизирующих затраты на оснащение узлов техническими средствами и их эксплуатацию при выполнении ограничений по оперативности, аппаратурной надежности выполнения задач управления, массе и энергопотреблению бортовой аппаратуры, загрузке узлов и др.
\end{abstract}

Ключевые слова: оптимизационно-имитационных подход, имитационное моделирование, алгоритм, модель, космические аппараты, сеть наземных пунктов управления 


\section{1. Введение}

Автоматизированные системы управления космическими аппаратами (АСУ КА) относятся к классу распределенных информационно-управляющих систем (ИУС). АСУ КА обладают рядом характерных особенностей, которые во многом усложняют задачу построения моделей и методов синтеза их структуры. Как правило, основными характеристиками, влияющими на выбор какоголибо пункта управления, являются динамические и стохастические характеристики, связанные с функционированием объектов управления. Это требует включения в процедуры синтеза их структуры моделей имитационного типа [1-5].

Анализ различных подходов к синтезу структуры подобных систем показал, что задачи синтеза могут быть разбиты на две группы. К первой относятся задачи, связанные с синтезом топологической структуры системы, состоящей в определении состава, территориального расположения и типа управляющих узлов на всех уровнях иерархии системы и каналов связи между ними.

Ко второй группе относятся проблемы синтеза функциональной структуры системы, т. е. распределение функций управления между узлами системы, включая объект управления и распределение технических средств по узлам системы.

Основываясь на оптимизационно-имитационном подходе применительно к анализу структур сложных систем, основное внимание уделяется потенциалу совместного использования в процессе синтеза оптимизационных и имитационных моделей, их рациональному взаимодействию в оптимизационно-имитационных процедурах, описывающих как состав и взаимосвязи структурных элементов системы, так и динамические и случайные аспекты их функционирования. Поэтому, основываясь на вышесказанном, применение анализируемых моделей в данной методологии значительно повысит качество принимаемых решений [6-9].

\section{2. Постановка проблем и задач: выявление плюсов и минусов модели}

Важной проблемой при создании систем управления, и в частности, систем управления космическими аппаратами с использованием автоматизации, является выбор их структуры, которая определяет состав элементов системы с соответствующими взаимосвязями и с учетом динамики их функционирования. Актуальными остаются вопросы постановки и формализации задач синтеза структур, разработки оптимизационных и имитационных моделей, а также построения на их основе процедур синтеза структуры автоматизированных систем управления космическими аппаратами. 
Задачи синтеза структуры сложных систем, учитывающие динамику функционирования элементов системы, только в простейших случаях могут быть решены аналитическими методами [10-13]. Использование же имитационных моделей позволяет учесть на этапе анализа и синтеза структуры не только статические взаимосвязи между элементами системы, но и динамические аспекты функционирования системы. Методы имитационного моделирования позволяют проанализировать только незначительное количество вариантов изучаемой системы и выбрать среди них в определенном смысле более приемлемый.

Ниже приводится перечень аргументов в пользу использования имитационного моделирования, а также некоторые минусы данной системы.

Преимущества:

- Разработка имитационной модели системы зачастую позволяет лучше понять реальную систему.

- В ходе моделирования возможно сокращение времени: годы практической эксплуатации реальной системы можно промоделировать в течение нескольких секунд или минут.

- Моделирование не требует прерывания текущей деятельности реальной системы.

- Имитационные модели носят намного более общий характер, чем математические модели; их можно использовать в тех случаях, когда для проведения стандартного математического анализа нет надлежащих условий.

- Моделирование обеспечивает более реалистичное воспроизведение системы, чем математический анализ.

- Моделирование возможно использовать для анализа переходных процессов, тогда как математические модели для этой цели не подходят.

- В настоящее время разработано множество стандартизованных моделей, охватывающих широкий спектр объектов реального мира.

Недостатки:

- Несмотря на то, что на разработку имитационной модели системы может уйти много времени и труда, нет никакой гарантии, что модель позволит получить ответы на интересующие вопросы.

- Нет никакого способа доказать, что работа модели полностью соответствует работе реальной системы. Моделирование связано с многочисленными повторениями 
последовательностей, которые основываются на генерации случайных чисел, имитирующих наступление тех или иных событий.

- В зависимости от системы, которую возможно моделировать, построение модели может занять от одного часа до 100 человеко-лет. Моделирование сложных систем может оказаться весьма дорогостоящей затеей и занять немало времени.

- Моделирование может быть менее точным, чем математический анализ, поскольку в его основу положена генерация случайных чисел.

- Для разработки и внедрения сложных моделей требуется довольно значительное компьютерное время.

\section{3. Методы исследования и полученные результаты}

Для решения проблем синтеза структуры распределенных АСУ КА на основе методологии синтеза структуры сложных систем, разработан комплекс взаимосвязанных моделей и алгоритмов и итеративные схемы их взаимодействия при синтезе топологической (пространственной) и функциональной структуры системы (рисунок 1) [14-18].

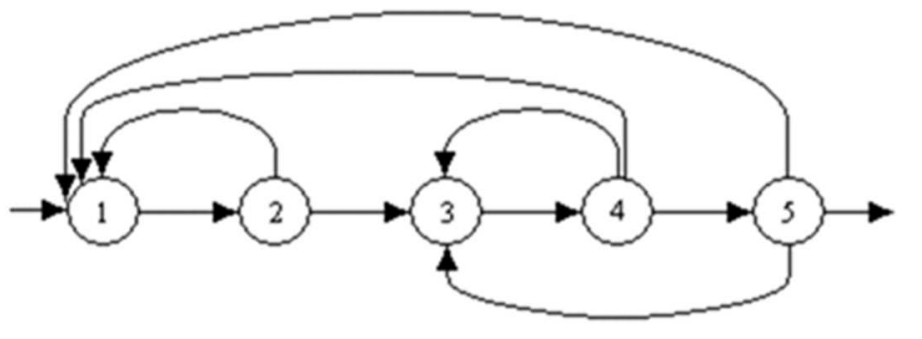

Рисунок 1. Схема взаимодействия процедур и алгоритмов при решении задачи выбора пунктов управления космическими аппаратами.

На первом этапе синтеза (блок 1) решается задача выбора состава и пространственного размещения управляющих узлов с учетом затрат на их организацию, доступность и глобальность управления различными классами КА.

Для определения алгоритмически заданных характеристик функционирования синтезированного варианта структуры системы предназначены имитационные модели взаимодействия элементов заданной топологической структуры (блок 2).

В блоках 3, 4 для выбранной совокупности пунктов управления и их взаимосвязей определяются варианты построения узлов и связей между ними, обеспечивающие повышение живучести системы, определяемой вероятностью выполнения системой функций управления с учетом возможных неблагоприятных внешних воздействий [9]. 
На этапе синтеза (блок 5) для найденного множества узлов управления и вариантов их реализации, работающих с объектами различных классов, и заданных функции управления по каждому классу объектов определяется оптимальное распределение задач взаимосвязанных подсистем по уровням и узлам системы и выбирается состав комплекса технических средств. Функции управления задаются в виде множества задач, выполняемых различными подсистемами (контурами) управления.

Для синтеза структур сложных систем разработан и успешно используется для решения практических задач структурного построения сложных систем оптимизационно-имитационный подход.

Оптимизационно-имитационный подход к синтезу структур сложных систем основан на совместном использовании в процессе поиска оптимального варианта структуры оптимизационных и имитационных моделей. Эти модели описывают как состав и взаимосвязи структурных элементов системы, так и динамические и стохастические аспекты их функционирования.

При этом возникает проблема рационального сочетания оптимизационных и имитационных моделей для синтеза структуры сложных систем. Это приводит к специфическим итеративным процедурам поиска рациональных вариантов структуры системы.

\section{4. Заключение}

При выборе структуры АСУ КА задачи формирования, учитывающие динамику функционирования элементов системы, только в простейших случаях могут быть решены аналитическими методами. Использование же оптимизационно-имитационных моделей позволяет учесть на этапе анализа и синтеза структуры не только статические взаимосвязи между элементами системы, но и динамические аспекты функционирования системы. Применение имитационных моделей дает множество преимуществ по сравнению с выполнением экспериментов над реальной системой и использованием других методов. Существует возможность модификации имитационной модели, по которой вновь производятся расчеты, а затем проводится сравнительный анализ новых результатов с полученными ранее. Методы имитационного моделирования, хотя и не приводят к получению оптимальных решений, как, например, методы линейного программирования, однако, позволяют выработать направления деятельности, приводящей к лучшим результатам. Но прежде, чем внедрять какой-либо из результатов, полученных по имитационной модели, в практику, необходимо произвести оценку ее надежности и, осуществив расчеты на более длительный период, получить репрезентативные 
характеристики. Обычно расчеты по имитационным моделям проводятся с помощью пакетов прикладных программ.

\section{Благодарности}

Работа выполнена в рамках государственного задания по теме № 0555-2021-0005. Работа выполнена в рамках государственного задания Минобрнауки России по теме «Концептуальное моделирование информационно-образовательной среды воспроизводства человеческого капитала в условиях цифровой экономики» (Шифр FNRN - E). Работа выполнена в рамках государственного задания Минобрнауки России по теме «Разработка новых методов автономной навигации космических аппаратов в космическом пространстве» (Шифр FNRN - S).

\section{Список литературы}

[1] Тюпкин, М.В. Аппаратно-информационные технологии управления космическими аппаратами / М.В. Тюпкин // Успехи соврем. естествознания. - 2006. - № 9. - С. 111-113.

[2] Карасева, М.В. Метапоисковая мультилингвистическая система / М.В. Карасева, И.Н. Карцан, П.В. Зеленков // Вестник Сибирского государственного аэрокосмического университета им. академика М.Ф. Решетнева. - 2007. - № 3(16). - С. 69-70.

[3] Царев, Р.Ю. Синтез и управление развитием кластерных структур АСУ космических систем / Р. Ю. Царев, Д.В. Капулин, А.В. Штарик, Е.Н. Штарик // Вестник СибГАУ. - 2012. - № 2(42). - С. $80-84$.

[4] Усольцев, А.А. Анализ методов структурно-параметрического описания объекта проектирования / А.А. Усольцев // Вестник НИИ СУВПТ. - 2003. - № 12. - С. 19-27.

[5] Клейнен, Дж. Статистические методы в имитационном моделировании / Дж. Клейнен. - М.: Статистика, 2008.

[6] Филиппов, В.А. Имитационное моделирование при синтезе структур сложных систем / В.А. Филиппов, А.Д. Цвиркун, В.К. Акинфиев. - Саратов: Изд-во Сарат. ун-та, 2003.

[7] Строгалев, В.П. Имитационное моделирование / В.П. Строгалев, И.О. Толкачева. - МГТУ им. Баумана, 2008. - 697-737 с.

[8] Елубаев, С.А. Имитационное моделирование основных компонентов системы управления движением космического аппарата / С.А. Елубаев, Н.К. Джамалов, К.А. Алипбаев, и др. // Вестник СибГАУ. - 2013. - №2(48).

[9] Лысенко, Л.Н. Концепция разработки программно-методического обеспечения комплекса имитационного моделирования условий функционирования орбитального сегмента 
глобальных навигационных и телекоммуникационных геостационарных спутниковых систем / Л.Н. Лысенко, В.В. Корянов, А.Г. Топорков // Инженерный журнал: наука и инновации. - 2015. - 5. - Режим доступа: http://engjournal.ru/catalog/arse/adb/1398.html.

[10] Карцан, И.Н. Мультиверсионное программное обеспечение бортового комплекса управления с генетическим алгоритмом / И.Н. Карцан // Решетневские чтения: материалы XXI международной научно-практической конференции (08-11 ноября 2017, г. Красноярск). Красноярск: СибГУ им. М.Ф. Решетнева. - 2017. - № 1. - С. 372-373.

[11] Тюпкин, М.В. Многоатрибутивные методы принятия решений в системном анализе / М.В. Тюпкин // Современные наукоемкие технологии. - 2007. - № 12. - С. 48-50.

[12] Филиппов, В. А. Оптимизационно-имитационные процедуры синтеза структур распределенных систем управления / В.А. Филиппов, А.Д. Цвиркун. - Саратов: Изд-во Сарат. ун-та, 2000.

[13] Kartsan, I.N. The hardware and software implementation of the adaptive platform for an onboard spacecraft control system / I.N. Kartsan, A.O. Zhukov, O.A. Platonov, S.V. Efremova // Journal of Physics: Conference Series. - 2019. - № 1399(3). - P. 033071.

[14] Карцан, И.Н. Модульно-иерархическое построение программного обеспечения при сетевом взаимодействии наземных пунктов / И.Н. Карцан, Л.М. Скопинцева, Д.Д. Дмитриев, С.В. Ефремова, В.В. Мосиенко // Актуальные проблемы авиации и космонавтики. - 2018. - T. 2. - № 4(14). - C. 151-154.

[15] Карцан, И.Н. Мультиверсионная модель программного обеспечения систем управления космическим аппаратом с ранжированием принятия решения / И.Н. Карцан, С.В. Ефремова // Сибирский аэрокосмический журнал. - 2021. - Т. 22. - № 1. - С. 32-46.

[16] Кобзарь, А.И. Прикладная математическая статистика. Для инженеров и научных работников / А.И. Кобзарь. М.: Физматлит, 2006. - 816 с.

[17] Скобелев, П.О. Мультиагентная система для исследования методов взаимодействия космических аппаратов дистанционного зондирования Земли / П.О. Скобелев, А.В. Соллогуб, А.В. Иващенко, Е.В. Симонова, М.Е. Степанов, А.В. Царев // Перспективные информационные технологии для авиации и космоса (ПИТ-2010). Труды Международной конференции с элементами научной школы для молодежи. Самара: СГАУ, 2010. - 226-230 c.

[18] Тюпкин, М.В. Проблема синтеза структуры АСУ космическими аппаратами / М.В. Тюпкин, Р.Ю. Царев // Фундаментальные исследования. - 2007. - №8. - С. 36-38. 